\title{
The X-Y factor: Females and males with urologic chronic pelvic pain syndrome present distinct clinical phenotypes
}

Gregory William Hosier; R. Christopher Doiron; Victoria Tolls; J. Curtis Nickel

Department of Urology, Queen’s University, Kingston, ON, Canada

Cite as: Can Urol Assoc J 2018 Feb. 23; Epub ahead of print. http://dx.doi.org/10.5489/cuaj.4798

Published online February 23, 2018

$* * *$

\section{Abstract}

Introduction: Urological chronic pelvic pain syndrome (UCPPS) in females is often attributed to the bladder (interstitial cystitis/bladder pain syndrome), while UCPPS in males is often attributed to the prostate (chronic prostatitis/chronic pelvic pain syndrome). However, there is increasing awareness that bladder pain plays a role in both males and females and the degree of overlap of clinical characteristics in males and females with UCPPS is not well known. Our objective was to compare clinical phenotypes of females and males with UCPPS.

Methods: We conducted a retrospective analysis of prospectively collected data from a singlecentre patient population presenting between 1998 and 2016 to our UCPPS clinic. Demographics, symptom scores, pain scales, retrospectively described clinical UPOINT (urinary, psychosocial, organ specific, infection, neurogenic, and tenderness) scoring, and presence of comorbid medical conditions were compared between females and males using comparative analyses.

Results: We identified 2007 subjects (1523 males, 484 females) with UCPPS. Females had increased prevalence of irritable bowel syndrome ( $25 \%$ vs. $11.2 \%$ ), chronic fatigue syndrome (13.6\% vs. $1.6 \%$ ), fibromyalgia ( $16.9 \%$ vs. $1.6 \%$ ), drug allergies ( $56.6 \%$ vs. $13.5 \%)$, diabetes ( $20.2 \%$ vs. $3.9 \%$ ), depression ( $31 \%$ vs. $18.4 \%$ ), and alcohol use ( $44.2 \%$ vs. $10.8 \%$ ) compared to males with UCPPS (all $\mathrm{p}<0.001$ ). In respect to UPOINT domains, females had a higher "total" (3.2 vs. 2.4), "urinary” (92.8\% vs. $67.6 \%)$, “organ-specific" ( $90.1 \%$ vs. $51.4 \%$ ), and "neurogenic" ( $44.7 \%$ vs. $30 \%$ ) prevalence compared to males (all p $<0.001$ ).

Conclusions: Females with UCPPS have greater prevalence of systemic disorders/symptoms and worse urinary symptoms than males with UCPPS. These findings demonstrate that females and males with UCPPS have distinct and different clinical phenotypes. 


\section{Introduction}

Urologic chronic pelvic pain syndrome (UCPPS) refers to chronic and often debilitating pain in the pelvis, prostate, bladder, and/or genitalia. ${ }^{1}$ UCPPS encompasses interstitial cystitis/bladder pain syndrome (IC/BPS) and chronic prostatitis/chronic pelvic pain syndrome (CP/CPPS). ${ }^{1}$ IC/BPS is defined as persistent or recurrent chronic pelvic pain, pressure or discomfort perceived to be related to the urinary bladder accompanied by at least one other urinary symptom such as urgency or frequency. ${ }^{2} \mathrm{CP} / \mathrm{CPPS}$ is defined as chronic genitourinary pain in the absence of uropathogenic bacteria localized to the prostate gland. ${ }^{3}$ Although UCPPS in males is often attributed to the prostate (CP/CPPS) and UCPPS in females is often attributed to the bladder (IC/BPS), there is increasing awareness that males may also have bladder involvement. ${ }^{4,5}$ In a population screening study, $48 \%$ of males screened positive for IC/BPS using a high specificity definition of IC/BPS and a further 18\% screened positive for both CP/CPPS and IC/BPS. ${ }^{4}$ This has led some to propose that IC/BPS and CP/CPPS may represent analogous conditions in females and males respectively. ${ }^{6-8}$

To date there have been limited studies comparing clinical characteristics of UCPPS in males and females. Using the MAPP database, Clemens et al. found variation in rates and severity of bladder symptoms among females and males with UCPPS. ${ }^{9}$ Also using the MAPP database, Lai and colleagues found that those who had a subtype of UCPPS with pain outside of the pelvis had higher rates of fibromyalgia, chronic fatigue syndrome, and irritable syndrome compared to those with pain limited to the pelvis. ${ }^{10}$ However, they did not compare rates of fibromyalgia, chronic fatigue syndrome, and irritable syndrome among females and males. UPOINT has been shown to be a useful clinical tool for phenotyping males with CP/CPPS ${ }^{11}$ and females with IC/BPS ${ }^{12}$ as well as guiding treatment decisions. Our objective was to compare the entire clinical phenotypes of males and females presenting with UCPPS to a single tertiary referral center by examining urinary and pain symptoms, UPOINT scores, and associated medical conditions. To further characterize sex-based phenotypic differences among patients with UCPPS, we compared a subgroup of males with IC/BPS to females with IC/BPS.

\section{Methods}

Participants and study design

This is a retrospective evaluation of a large, prospective, clinical quality assurance database of patients with IC/BPS and CP/CPPS treated at our outpatient clinic by a single urologist (JCN) between 1998 and 2016. Our IC/BPS patient sample has been described in previous publications. ${ }^{13}$ Briefly, patients were diagnosed with IC/BPS on the basis of chronic (>6 months) pelvic pain, pressure or discomfort perceived to be related to the urinary bladder accompanied by at least 1 other urinary symptom such as urgency or frequency. Exclusion criteria included positive urine culture or cytology. All other diseases that could cause pelvic symptoms were excluded with our standardized history and physical examination, which included a pelvic examination. Our male CP/CPPS patient sample has also been described in previous 
publications. ${ }^{14,15}$ These included male patients referred by both primary care physicians or urologists to the Queen's University Prostatitis Research Center who have fulfilled the National Institutes of Health/National Institute for Diabetes and Digestive and Kidney Diseases criteria for a diagnosis of CP/CPPS. ${ }^{16}$ Briefly these would include men with pelvic pain for 3 of the previous 6 months, with or without voiding symptoms, and with no evidence of uropathogenic bacterial infection.

This retrospective study was done under ongoing institutional review board approval for continued quality assurance with all patient data de-identified before analysis.

\section{Measures}

Data on patient demographics, symptom duration, urinary frequency scores (0-5), pain frequency scores (0-5), pain severity (0-10), clinical UPOINT scoring (retrospectively determined on patients assessed prior to 2009), and the presence or absence of co-morbid medical conditions (fibromyalgia, irritable bowel syndrome, chronic fatigue syndrome, diabetes, depression, alcohol use, food/drug allergies, and hypertension) were collected through initial evaluations at the IC/BPS and CP/CPPS outpatient clinics. IBS diagnosis was made based on the Rome III criteria. Patient self-report assessed presence of the remaining conditions. UPOINT descriptions have been published previously. ${ }^{11,12}$

\section{Data analysis}

Statistical analysis was completed using the Microsoft Excel 2010 Data Analysis package and the Social Science Statistics (http://www.socscistatistics.com/). In all cases statistical analysis was done between male and female cohorts. Categorical data, including UPOINT domains and the presence or absence of co-morbid medical conditions, were analyzed using the 2-tailed z-test for population proportions. Other quantitative data, age, symptom duration and all questionnaire scores were analyzed using the 2-tailed t-test and assuming unequal variances. All results considered statistically significant at $\mathrm{p}<0.05$.

\section{Results}

We identified 2007 subjects (1523 males; 484 females) with UCPPS. Of the 1523 male patients, 26 had IC/BPS and 7 had Hunner lesions identified on cystoscopy. Of the 484 female patients with IC/BPS, 41 had Hunner lesions identified on cystoscopy. Table 1 shows a comparison of demographics, symptom scores, UPOINT scores, and medical co-morbidities. Females had statistically significantly longer symptom duration (at time of referral to the tertiary urology pain clinic) compared to males ( 10.7 vs. 6.75 years, $\mathrm{p}<0.001)$. While females reported significantly higher scores for urinary frequency $(4.0 \pm 1.3$ vs. $2.7 \pm 1.7, \mathrm{p}<0.001)$ and pain frequency (3.2 \pm 1.5 vs. $3.0 \pm 1.5, p<0.001$ ), males reported higher pain intensity scores ( $4.6 \pm 2.7$ vs. $4.1 \pm 3.6$, $\mathrm{p}=0.006)$. 
When comparing prevalence of UPOINT domains, females had a higher total prevalence compared to males ( 3.2 vs. $2.4, \mathrm{p}<0.001$ ). Specific UPOINT domains that were more prevalent in females were "urinary” ( $92.8 \%$ vs. $67.6 \%$, p $<0.001)$, “organ specific” ( $90.1 \%$ vs. $51.4 \%$, $\mathrm{p}<0.001)$, and "neurogenic" ( $44.7 \%$ vs. $30 \%$, $\mathrm{p}<0.001)$. Females had a lower prevalence of UPOINT "tenderness" ( $42.7 \%$ vs. $48.6 \%, \mathrm{p}=0.02)$ and "infection" ( $21.1 \%$ vs. $32.8 \%, \mathrm{p}<0.001)$ domains compared to males.

There were notable differences in the presence of co-morbid medical conditions as well. Females were found to have a higher prevalence of fibromyalgia ( $16.9 \%$ vs. $1.5 \%, \mathrm{p}<0.001)$, chronic fatigue syndrome ( $13.6 \%$ vs. $1.6 \%, \mathrm{p}<0.001)$, irritable bowel syndrome ( $25 \%$ vs. $11.2 \%$, $\mathrm{p}<0.001$ ), drug allergies ( $56.6 \%$ vs. $13.5 \%$, $\mathrm{p}<0.001$ ), diabetes ( $20.2 \%$ vs. $3.9 \%, \mathrm{p}<0.001$ ), depression ( $31 \%$ vs. $18.4 \%, \mathrm{p}<0.001)$, and alcohol use $(44.2 \%$ vs. $10.8 \%, \mathrm{p}<0.001)$ compared to the male cohort.

A subgroup analysis of males diagnosed with IC/BPS compared to females with IC/BPS was performed (Table 2). Females with IC/BPS were younger than males with IC/BPS at time of presentation ( 45.7 vs. 58.5 years, $\mathrm{p}<0.001$ ). In respect to UPOINT domain prevalence, the female IC/BPS patients had higher "infection" $(21.1 \%$ vs. $0 \%, \mathrm{p}=0.009)$ and "neurogenic" $(44.7 \%$ vs. $23.1 \%, \mathrm{p}=0.03$ ) domain prevalence compared to males with IC/BPS. Furthermore, the females had a higher prevalence of irritable bowel syndrome ( $25 \%$ vs. $3.8 \%, \mathrm{p}=0.01)$ and drug allergies ( $56.6 \%$ vs. $30.8 \%, \mathrm{p}=0.01$ ) compared to males with IC/BPS. Due to the low number of males with IC Hunner lesions ( $\mathrm{n}=7$ ), we did not compare males and females with IC Hunner lesion phenotype.

\section{Discussion}

As there is increasing awareness that UCPPS in males may relate not only to the prostate, more have argued that IC/BPS and CP/CPPS may represent different manifestations of the same disease process. ${ }^{6-8}$ However, data comparing clinical characteristics of UCPPS in males and females has been lacking. In this retrospective analysis of prospectively collected data on a large, single-center patient population, we show several important differences including a much higher prevalence of co-morbid medical conditions, greater prevalence of UPOINT domains, and worse urologic symptoms in females compared to males with UCPPS.

Although it is well established that males and females with UCPPS have increased prevalence of co-morbid functional/systemic symptoms compared to healthy controls ${ }^{17-19}$, there have been no studies to date directly comparing the presence of co-morbid functional/systemic conditions among males and females with UCPPS. We found that females had increased prevalence of irritable bowel syndrome ( $25 \%$ vs. $11.2 \%$ ), chronic fatigue syndrome (13.6\% vs. $1.6 \%$ ), and fibromyalgia (16.9 vs. $1.6 \%$ ) compared to males with UCPPS. Females also had increased prevalence of drug allergies ( $56.6 \%$ vs. $13.5 \%$ ), diabetes (20.2\% vs. $3.9 \%$ ), depression ( $31 \%$ vs. $18.4 \%$ ), and alcohol use ( $44.2 \%$ vs. $10.8 \%$ ), compared to males with UCPPS. Females in the general population are known to have increased prevalence of fibromyalgia, chronic fatigue syndrome, and irritable bowel syndrome compared to males with a female to male ratio 
of approximately $6: 1^{20}, 2: 1^{21}$, and 3:122 respectively. We found that females in our study with UCPPS had increased prevalence of fibromyalgia, chronic fatigue syndrome, and irritable bowel syndrome beyond that expected in non-UCPP populations with a female to male ratio of 11:1, 9:1, and 15:1 respectively. There is increasing awareness that patients with pain symptoms outside of the pelvis may represent a different clinical phenotype than those with pain limited to the pelvis. ${ }^{10,19,23}$ Patients with pain outside of the pelvis are hypothesized to have increased sensitization of central pain processing pathways leading to higher co-occurrence of other systemic pain conditions, such as irritable bowel syndrome, chronic fatigue syndrome, and fibromyalgia, while, in patients with pain limited to the bladder, pain has been hypothesized to occur through visceral nerve abnormalities involving only the bladder. ${ }^{23}$ Lai and colleagues found that those with pelvic pain and beyond phenotype had higher rates of fibromyalgia, chronic fatigue syndrome, and irritable syndrome compared to those with pain limited to the pelvis. ${ }^{10}$ However, they did not compare rates of fibromyalgia, chronic fatigue syndrome, and irritable syndrome between females and males. Our results indicate that gender itself may represent a distinct clinical phenotype in which females have greater prevalence of systemic pain/neural conditions. Hypersensitivity of peripheral and central pain processing pathways has been hypothesized to be a sex-biased consequence of chronic stress in females. ${ }^{24}$ This may underlie our findings of higher prevalence of somatic symptoms in females with UCPPS compared to males.

Interestingly, we found that females with UCPPS had higher rates of alcohol use compared to males with UCPPS whereas the opposite is true in the general population. ${ }^{25}$ This may be related to higher rates of depression and psychological stress in females compared to males with UCPPS, which have been associated with alcohol use. ${ }^{26}$ Prior studies have reported decreased rates of diabetes in patients with IC/BPS, however, in our study there was a significantly higher rate of diabetes in our female UCPPS population compared to rates reported in the general population (20\% vs. $6 \%$ ). ${ }^{27}$ The reason for this discrepancy is unclear.

UPOINT has been shown to be a useful clinical tool for phenotyping males with $\mathrm{CP} / \mathrm{CPPS}^{11}$ and females with IC/BPS ${ }^{12}$ as well as guiding treatment decisions, however no study to date has directly compared prevalence of UPOINT domains among males and females with UCPPS. Using UPOINT, we found an increased prevalence of "urinary," "organ specific," and "neurogenic" domains in females compared to males with UCPP, and increased "infection" and "tenderness" domains in males compared to females. The prevalence of UPOINT domains for males and females in the current study were similar to previous studies. ${ }^{11,12}$ As the criteria for diagnosis of IC/BPS in the current study included perceived bladder pain and the presence of at least one urinary symptom, the "urinary" and "organ specific" domains would be expected to be more prevalent based on diagnostic criteria alone.

While UPOINT domain prevalence between females with IC/BPS and the subgroup of males with IC/BPS were more similar, females with IC/BPS still had increased prevalence of "neurogenic" domains compared to males with IC/BPS. As far as we know, we are the first 
group to compare clinical phenotypes of females with IC/BPS and males specifically with IC/BPS. This is an important finding that indicates that sex-based phenotypic differences reported here and elsewhere cannot only be attributed to involvement of the prostate in males.

Similar to our findings of increased urinary and pain frequency among females compared to males with UCPPS, Clemens et al. found that females with IC/BPS had significantly worse urgency, frequency, and nocturia. ${ }^{9}$ In their prospective study comparing 233 females to 191 males, females reported higher scores on ICSI, ICPI, and AUASI, compared to males with CP/CPPS. ${ }^{9}$ In a large prospective survey study of 981 females and 1768 males, Marszalek et al. also found that women had a greater prevalence of storage urinary symptoms as measured on the CPSI and IPSS, compared to males. ${ }^{28}$

The findings of the current study have implications for our understanding and management of UCPPS in males and females. Different symptom patterns and clinical phenotypes suggest different etiologies and pathogenic pathways between the sexes. There is evidence that therapy directed at clinical UPOINT phenotypes leads to increased treatment success for IC/BPS ${ }^{13}$ and CP/CPPS ${ }^{11}$. It is likely that therapies directed at "urinary" (antimuscarinics, pyridium, bladder retraining), "organ specific" (pyridium, intravesical GAGs, DMSO, lidocaine, pentosan polysulfate sodium, quercetin) and "neurogenic" (amitriptyline, gabapentinoids, system specific therapies, specialist referral for systemic disorders) domains may be more effective overall in the female UCPPS population compared to males. In contrast, therapies directed at the "infection" (antimicrobials) and "tenderness" (pelvic floor physiotherapy, muscle relaxants, trigger point injections) domains may be more effective in the male UCPPS population compared to females. That being said, an individualized treatment approach should be taken based on each patient's unique clinical phenotype.

Limitations of the current study include the retrospective analysis (though data was collected prospectively). Patient self-report was used for all conditions other than irritable bowel syndrome allowing for the possibility that prevalence would differ using specific diagnostic criteria. Additionally, this was a single-centre study, which could limit external validity. Strengths of our study include the large study population, prospective data collection, and our use of Rome III diagnostic criteria for irritable bowel syndrome.

\section{Conclusions}

Females with UCPPS have worse urinary symptoms and greater prevalence of systemic disorders/symptoms including irritable bowel syndrome, chronic fatigue, and fibromyalgia, as well as increased prevalence of the UPOINT "urinary," "organ specific," and "neurogenic" domains compared to males with UCPP. Males exhibited increased prevalence of the "infection" and "tenderness" domains. These findings demonstrate distinct male and female phenotypes in patients with UCPPS. 


\section{References}

1. Clemens JQ, Mullins C, Kusek JW, et al. The MAPP research network: a novel study of urologic chronic pelvic pain syndromes. BMC Urol 2014;14:57-63.

2. Doggweiler R, Whitmore KE, Meijlink JM, et al. A standard for terminology in chronic pelvic pain syndromes: A report from the chronic pelvic pain working group of the international continence society. Neurourol Urodyn 2016.

3. Nickel JC, Nyberg LM, Hennenfent M. Research guidelines for chronic prostatitis: consensus report from the First National Institutes of Health International Prostatitis Collaborative Network. Urology1999;54:229-33.

4. Suskind AM, Berry SH, Ewing BA, et al. The prevalence and overlap of interstitial cystitis/bladder pain syndrome and chronic prostatitis/chronic pelvic pain syndrome in men: Results of the rand interstitial cystitis epidemiology male study. J Urol 2013;189(1):141-5.

5. Clemens JQ, Meenan RT, Rosetti MCO, et al. Prevalence and incidence of interstitial cystitis in a managed care population J Urol 2005;173:98-102.

6. Moldwin RM. Similarities between interstitial cystitis and male chronic pelvic pain syndrome. Curr Urol Rep 2002;3:313-8.

7. Pontari MA. Chronic prostatitis/chronic pelvic pain syndrome and interstitial cystitis: are they related? Curr Urol Rep 2006;7:329-34.

8. Forrest JB, Nickel JC, Moldwin RM. Chronic Prostatitis/Chronic Pelvic Pain Syndrome and Male Interstitial Cystitis: Enigmas and Opportunities. Urology 2007;69:S60-3.

9. Clemens JQ, Clauw DJ, Kreder K, et al. Comparison of baseline urologic symptoms in men and women in the multidisciplinary approach to the study of chronic pelvic pain (Mapp) research cohort. J Urol 2014;193:1554-8.

10. Lai HH, Jemielita T, Sutcliffe S, et al. Characterization of whole body pain in urological chronic pelvic pain syndrome at baseline: A MAPP Research Network Study. J Urol 2017;110. http://www.ncbi.nlm.nih.gov/pubmed/28373134

11. Shoskes DA, Nickel JC, Rackley RR, et al. Clinical phenotyping in chronic prostatitis/chronic pelvic pain syndrome and interstitial cystitis: a management strategy for urologic chronic pelvic pain syndromes. Prostate Cancer Prostatic Dis 2009;12:177-83.

12. Nickel JC, Shoskes D, Irvine-Bird K. Clinical Phenotyping of Women With Interstitial Cystitis/Painful Bladder Syndrome: A Key to Classification and Potentially Improved Management. J Urol 2009;182:155-60.

13. Nickel JC, Irvine-Bird K, Jianbo L, et al. Phenotype-directed management of interstitial cystitis/bladder pain syndrome. Urology 2014;84:175-9.

14. Nickel JC, Downey J, Ardern D, et al. Failure of monotherapy strategy for the treatment of difficult chronic prostatitis/chronic pelvic pain syndrome patients. J Urol 2004;172:551-554. 
15. Ginting JV, Tripp DA and Nickel JC. Self-reported spousal support modifies the negative impact of pain on disability in men with chronic prostatitis/chronic pelvic pain syndrome. Urology 2011;78:1136-1141.

16. Krieger JN, Nyberg L, and Nickel JC. NIH consensus definition and classification of prostatitis. JAMA, 1999; 282: 236-237.

17. Naliboff BD, Stephens AJ, Afari N, et al. Widespread psychosocial difficulties in men and women with urologic chronic pelvic pain syndromes: Case-control findings from the multidisciplinary approach to the study of chronic pelvic pain research network. Urology 2015;85:1319-27.

18. Nickel JC, Tripp DA, Pontari M, et al. Psychosocial phenotyping in women with interstitial cystitis/painful bladder syndrome: A case control study. J Urol 2010;183:167-72.

19. Nickel JC, Tripp DA, Carr LK, et al. Clinical and psychological parameters associated with pain pattern phenotypes in women with interstitial cystitis/bladder pain syndrome. J Urol 2015;193:138-44.

20. Jones GT, Atzeni F, Beasley M, et al. The prevalence of fibromyalgia in the general population: a comparison of the American College of Rheumatology 1990, 2010, and modified 2010 classification criteria. Arthritis Rheumatol 2015;67:568-75.

21. Bakken IJ, Tveito K, Gunnes N, et al. Two age peaks in the incidence of chronic fatigue syndrome/myalgic encephalomyelitis: a population-based registry study from Norway 20082012. BMC Med 2014;12:167.

22. Canavan C, West J, Card T. The epidemiology of irritable bowel syndrome. Clin Epidemiol 2014;6:71-80.

23. Tripp DA, Nickel JC, Wong J, et al. Mapping of Pain Phenotypes in Female Patients with Bladder Pain Syndrome/Interstitial Cystitis and Controls. Eur Urol 2012;62:1188-94. http://www.ncbi.nlm.nih.gov/pubmed/22633363

24. Mayer EA, Berman S, Chang L, et al. Sex-based differences in gastrointestinal pain. Eur J Pain 2004;8:451-63. http://www.ncbi.nlm.nih.gov/pubmed/15324776

25. Wilsnack RW, Wilsnack SC, Kristjanson AF, et al. Gender and alcohol consumption: patterns from the multinational GENACIS project. Addiction 2009;104:1487-500. http://www.ncbi.nlm.nih.gov/pubmed/19686518

26. Hasin DS, Stinson FS, Ogburn E, et al. Prevalence, Correlates, Disability, and Comorbidity of DSM-IV Alcohol Abuse and Dependence in the United States. Arch Gen Psychiatry 2007;64:830. http://www.ncbi.nlm.nih.gov/pubmed/17606817

27. Warren JW, Howard FM, Cross RK, et al. Antecedent Nonbladder Syndromes in CaseControl Study of Interstitial Cystitis/Painful Bladder Syndrome. Urology 2009;73:52-7. http://www.ncbi.nlm.nih.gov/pubmed/18995888

28. Marszalek M, Wehrberger C, Temml C, et al. Chronic pelvic pain and lower urinary tract symptoms in both sexes: Analysis of 2749 participants of an urban health screening project. Eur Urol 2009;55:499-508. 
Figures and Tables

\begin{tabular}{|c|c|c|c|c|c|}
\hline & $\mathbf{n}$ & Female & $\mathbf{n}$ & Male & $\mathbf{p}$ \\
\hline \multicolumn{6}{|l|}{ Age and symptoms, mean \pm SD } \\
\hline Age at presentation (years) & 484 & $45.7 \pm 17.4$ & 1523 & $45.1 \pm 13.5$ & 0.47 \\
\hline Symptom duration (years) & 367 & $10.7 \pm 11.7$ & 1238 & $6.75 \pm 8.6$ & $<0.001$ \\
\hline Urinary frequency (0-5) & 466 & $4.0 \pm 1.3$ & 1431 & $2.7 \pm 1.7$ & $<0.001$ \\
\hline Pain frequency (0-5) & 466 & $3.2 \pm 1.5$ & 1431 & $3.0 \pm 1.5$ & $<0.001$ \\
\hline Pain intensity (0-10) & 464 & $4.1 \pm 3.6$ & 1431 & $4.6 \pm 2.7$ & 0.006 \\
\hline \multicolumn{6}{|l|}{ UPOINT } \\
\hline Total (0-6), mean \pm SD & 483 & $3.2 \pm 1.4$ & 1516 & $2.4 \pm 1.2$ & $<0.001$ \\
\hline Urinary, n (\%) & 483 & $448(92.8)$ & 1523 & $1029(67.6)$ & $<0.001$ \\
\hline Psychosocial, n (\%) & 483 & $153(31.7)$ & 1523 & $427(28.0)$ & 0.12 \\
\hline Organ specific, n (\%) & 483 & 435 (90.1) & 1523 & $783(51.4)$ & $<0.001$ \\
\hline Infection, n (\%) & 483 & $102(21.1)$ & 1523 & $499(32.8)$ & $<0.001$ \\
\hline Neurogenic, n (\%) & 483 & $216(44.7)$ & 1523 & $457(30.0)$ & $<0.001$ \\
\hline Tenderness, n (\%) & 483 & $206(42.7)$ & 1523 & $740(48.6)$ & 0.02 \\
\hline \multicolumn{6}{|l|}{ Medical history, n (\%) } \\
\hline Fibromyalgia & 484 & 82 (16.9) & 1523 & $23(1.5)$ & $<0.001$ \\
\hline Chronic fatigue syndrome & 484 & $66(13.6)$ & 1523 & $25(1.6)$ & $<0.001$ \\
\hline Irritable bowel syndrome & 484 & $121(25)$ & 1523 & $170(11.2)$ & $<0.001$ \\
\hline Drug allergies & 484 & $274(56.6)$ & 1523 & $205(13.5)$ & $<0.001$ \\
\hline Food allergies & 484 & $67(13.8)$ & 1523 & $174(11.4)$ & 0.15 \\
\hline Diabetes & 484 & $98(20.2)$ & 1523 & $59(3.9)$ & $<0.001$ \\
\hline Depression & 484 & $150(31)$ & 1523 & $280(18.4)$ & $<0.001$ \\
\hline Hypertension & 484 & $85(17.6)$ & 1523 & $844(55.4)$ & $<0.001$ \\
\hline Alcohol use & 484 & $214(44.2)$ & 1523 & $165(10.8)$ & $<0.001$ \\
\hline
\end{tabular}

SD: standard deviation. 


\begin{tabular}{|l|c|c|c|c|c|}
\hline \multicolumn{7}{|c|}{ Table 2. Comparison of females with IC/BPS to a subset of males with IC/BPS } \\
\hline & $\mathbf{n}$ & Female & $\mathbf{n}$ & Male & p \\
\hline Age and symptoms, mean \pm SD & & & & & \\
\hline Age at presentation (years) & 484 & $45.7 \pm 17.4$ & 26 & $58.5 \pm 13.0$ & $<0.001$ \\
\hline Symptom duration (years) & 367 & $10.7 \pm 11.7$ & 22 & $7.6 \pm 6.8$ & 0.55 \\
\hline Urinary frequency (0-5) & 466 & $4.0 \pm 1.3$ & 23 & $4.1 \pm 1.5$ & 0.66 \\
\hline Pain frequency (0-5) & 466 & $3.2 \pm 1.5$ & 23 & $2.8 \pm 1.7$ & 0.21 \\
\hline Pain intensity (0-10) & 464 & $4.1 \pm 3.6$ & 26 & $4.2 \pm 3.9$ & 0.94 \\
\hline UPOINT & & & & & \\
\hline Total (0-6), mean \pm SD & 483 & $3.2 \pm 1.4$ & 26 & $2.5 \pm 0.9$ & $<0.001$ \\
\hline Urinary, n (\%) & 483 & $448(92.8)$ & 26 & $25(96.2)$ & 0.51 \\
\hline Psychosocial, n (\%) & 483 & $153(31.7)$ & 26 & $6(23.1)$ & 0.12 \\
\hline Organ specific, n (\%) & 483 & $435(90.1)$ & 26 & $24(92.3)$ & 0.71 \\
\hline Infection, n (\%) & 483 & $102(21.1)$ & 26 & $0(0)$ & 0.008 \\
\hline Neurogenic, n (\%) & 483 & $216(44.7)$ & 26 & $6(23.1)$ & 0.03 \\
\hline Tenderness, n (\%) & 483 & $206(42.7)$ & 26 & $4(15.4)$ & 0.06 \\
\hline Medical history, n (\%) & & & & & \\
\hline Fibromyalgia & 484 & $82(16.9)$ & 26 & $1(3.8)$ & 0.078 \\
\hline Chronic fatigue syndrome & 484 & $66(13.6)$ & 26 & $1(3.8)$ & 0.14 \\
\hline Irritable bowel syndrome & 484 & $121(25)$ & 26 & $1(3.8)$ & 0.014 \\
\hline Drug allergies & 484 & $274(56.6)$ & 26 & $8(30.8)$ & 0.01 \\
\hline Food allergies & 484 & $67(13.8)$ & 26 & $2(7.7)$ & 0.37 \\
\hline Diabetes & 484 & $98(20.2)$ & 26 & $4(15.4)$ & 0.55 \\
\hline Depression & 484 & $150(31)$ & 26 & $4(15.4)$ & 0.09 \\
\hline Hypertension & 484 & $85(17.6)$ & 26 & $7(26.9)$ & 0.23 \\
\hline Alcohol use & 484 & $214(44.2)$ & 26 & $11(42.3)$ & 0.85 \\
\hline C/BPS: inn & & & \\
\hline
\end{tabular}

IC/BPS: interstitial cystitis/bladder pain syndrome; SD: standard deviation. 